\title{
Design, analysis and fabrication of conceptual innovative hand tool
}

\begin{abstract}
Dioscorea hispida is a poisonous plant normally found in forest where scientific studies have shown that its tuber contains toxic poison and can be consumed after its poison is removed. Traditionally the tubers were placed in flowing river water for several days. In the harvesting aspect, an innovative hand tool were conceptually designed to replace the traditional hoe or ñcangkulò. Using IMADA digital force gauge to measure the force required for pulling out the tuber and reverse engineering methods, an innovative hand tool were designed and developed. The results from experiments showed that the force required for harvesting the Dioscorea hispida tuber is found significant with its weight versus regression squared (R2) of 0.86. The information of optimum force required from field experiment is used to model the simulation and practicality in Computer Aided Design (CAD) environment system. The finite element on stress distribution on selected material of hand tool was simulated by uploading the material characteristic on simulation program embedded in Solidworks software. The end result of the simulation is based on visualization of analysis in Solidworks while producing the hand tool for designing and fabrication from material which is lighter and stronger.
\end{abstract}

Keyword: Digital force gauge; Dioscorea hispida; Finite element; Modeling; Simulation 